\title{
Water on graphene: Hydrophobicity and dipole moment using density functional theory
}

\author{
O. Leenaerts, ${ }^{1, *}$ B. Partoens, ${ }^{1, \dagger}$ and F. M. Peeters ${ }^{1,2, \ddagger}$ \\ ${ }^{1}$ Departement Fysica, Universiteit Antwerpen, Groenenborgerlaan 171, B-2020 Antwerpen, Belgium \\ ${ }^{2}$ Departamento de Física, Universidade Federal do Ceará, Caixa Postal 6030, Campus do Pici, 60455-760 Fortaleza, CE, Brazil
}

(Received 24 March 2009; published 29 June 2009)

\begin{abstract}
We apply density-functional theory to study the adsorption of water clusters on the surface of a graphene sheet and find i) graphene is highly hydrophobic and ii) adsorbed water has very little effect on the electronic structure of graphene. A single water cluster on graphene has a very small average dipole moment which is in contrast with an ice layer that exhibits a strong dipole moment.
\end{abstract}

DOI: $10.1103 /$ PhysRevB.79.235440

PACS number(s): 73.20.Hb, 68.43.Bc, 81.05.Uw

\section{INTRODUCTION}

Graphene ${ }^{1}$ is predicted to be a very promising material for future electronics due to several useful properties such as the high mobility of the charge carriers and high crystal quality. Because of its essentially two-dimensional nature, graphene is always completely exposed to the environment and it is, therefore, important to have a good knowledge of any influence of this environment on the electronic properties. Graphene is completely surface which is advantageous when one wants to use graphene as a sensitive gas sensor. ${ }^{2}$ But on the other hand, the close contact of graphene with its environment is often a source of noise that makes different graphene samples behave in a quantitatively different manner. For example, the mobility and the density of charge carriers depend crucially on the environment. Important influences from the environment are the nature of the underlying substrate, which supports graphene, and the presence of molecules that adsorb on the exposed graphene surface or edges. ${ }^{3-10}$

In this paper we investigate the adsorption of water clusters on graphene. This is important because water is almost always present in the environment of any graphene device. Although it has been shown that a single water molecule does not bind strongly on a graphene surface, ${ }^{5}$ the large dipole moment associated with water molecules might still be able to modify the electronic properties, e.g., its mobility. Here we will investigate different systems consisting of small water clusters and the limit of ice on freestanding graphene sheets. We look for the optimal orientation of these clusters with respect to the graphene sheet and use the density-functional theory (DFT) to calculate different properties of the adsorbed clusters that might change the electronic properties of graphene.

This paper is organized as follows. In Sec. II we present our numerical approach. Section III contains our numerical results for the geometry of the water clusters, its binding energy on graphene, its charge transfer (CT) to graphene, and its dipole moment. We also present results in the limit of a complete wetting, namely, a single ice layer on top of graphene. Our conclusions are given in Sec. IV.

\section{COMPUTATIONAL DETAILS}

We study the interaction of graphene with water clusters using DFT within the generalized gradient approximation of
Perdew-Burke-Ernzerhof (PBE-GGA), ${ }^{11}$ which has been shown to give good results for $\mathrm{H}_{2} \mathrm{O}$ hydrogen bonding. ${ }^{12}$ Previous calculations of clustered water molecules inside and on top of carbon nanotubes ${ }^{13,14}$ suggest that the interaction between water molecules and graphenelike structures is described reasonably well, although the usual DFT calculations do not take explicitly into account the van der Waals ( $\mathrm{vdW}$ ) interactions. This is due to the polar nature of the water molecules which is responsible for the largest part of the $\mathrm{vdW}$ interaction in these systems and which is included in the calculations. We make use of a projected augmented wave-basis set with a plane-wave cutoff energy of 20 Hartree and perform the calculations with the ABINIT code. ${ }^{15,16}$ The simulated graphene sheet consists of a $4 \times 4$ supercell with a distance of $16 \AA$ between adjacent layers. A $6 \times 6 \times 1$ Monkhorst-Pack (MP) grid $^{17}$ is used for the sampling of the Brillouin zone. When studying ice on graphene, we use a $(\sqrt{3} \times \sqrt{3}) \mathrm{R}^{2} 0^{\circ}$ supercell $^{4}$ and a $15 \times 15 \times 1$ MP grid.

\section{RESULTS}

We examine the adsorption of $\mathrm{H}_{2} \mathrm{O}$ on graphene of clusters containing up to five water molecules, and a full monolayer of ice for different possible directions of the water molecules. The energetic favorable adsorption site for a single molecule [see Fig. 1(a)] was determined previously ${ }^{5}$ and, therefore, there is no need to vary it here. Moreover, it was also shown that the adsorption site has only a minor influence on the characteristic properties of the adsorption process. ${ }^{5}$ Because the binding energies between the $\mathrm{H}_{2} \mathrm{O}$ molecules in a water cluster are more than an order of magnitude larger than the adsorption energies associated with the binding of the clusters to graphene (see below), we consider only those water clusters that have the lowest energy and vary the orientation of the cluster with respect to the graphene crystallographic axes.

\section{A. Cluster geometry}

We first look for the most stable geometries of freestanding planar water clusters with up to five molecules, based on earlier investigations of freestanding water clusters ${ }^{18,19}$ and water clusters on graphite. ${ }^{20}$ In accordance with those earlier studies, we find that (except for the water dimer and monomer) the optimized clusters consist of rings of water mol- 

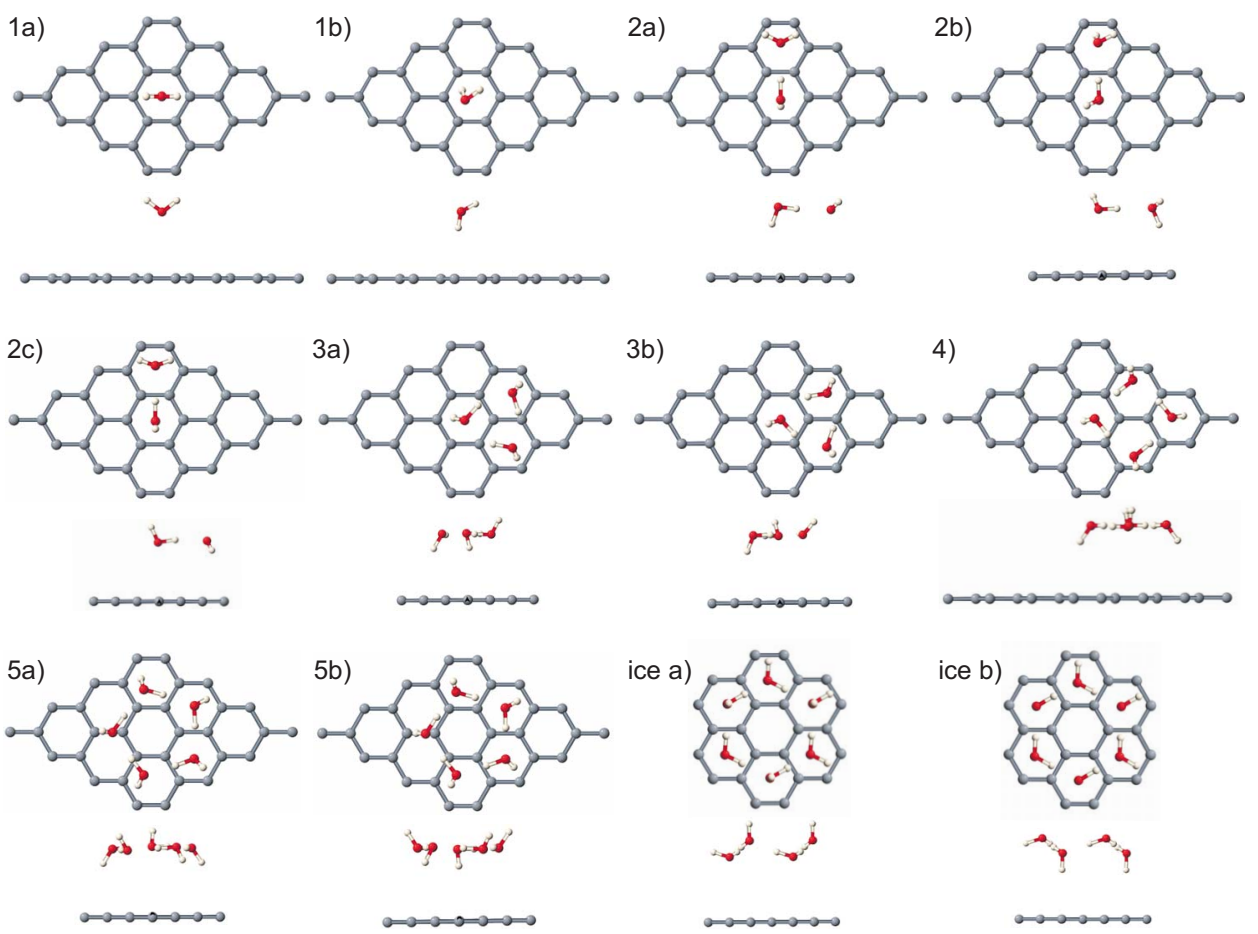

ecules formed in such a way that every water molecule uses one hydrogen atom to form a hydrogen bond with its neighbors. The other hydrogen atoms are pointing out of the plane formed by the ring, and alternate their direction as much as possible in order to minimize the interaction between hydrogen atoms of neighboring molecules.

After obtaining the relaxed geometries, we put these optimized clusters on a perfect graphene sheet and let the system relax (see Fig. 1). As a starting point for the relaxation, we use two orientations with respect to the graphene surface for the monomer and three for the dimer. More orientations that are meaningful are possible for the monomer, but these have already been studied elsewhere ${ }^{5}$ and, therefore, we do not need to consider them here. For the ring-shaped clusters, the starting point for relaxation is such that the ring is more or less parallel to the surface. We use the notations introduced in Fig. 1 for the different clusters with their various orientations.

The orientation and the shape of the clusters do not change much after relaxation but the clusters do tend to become more flat. For example, a freestanding cluster with five molecules forms a pentagon that is slightly puckered, ${ }^{19}$ but this puckering disappears when the water pentamer adsorbs on graphene. These results are different from recent theoretical results where it was proposed that the adsorption of water clusters on graphene occurs through a 'cluster link' consisting of a single water molecule. ${ }^{21}$ As we will show below, bonding through a single molecule seems surprising for two reasons: (i) the binding energy between molecules in a water cluster is much larger than the adsorption energy between the water cluster and graphene, which makes a deformation of the water cluster to form a cluster link unlikely and (ii) the adsorption energy of a water cluster is larger than the adsorption energy of a single molecule, implying that the cluster will try to adsorb as many molecules as possible (without
FIG. 1. (Color online) View from the top and the side of all the different water clusters on top of graphene that were investigated in our simulations with their various orientations. changing the geometry of the cluster too much). We also studied a monolayer of ice $\mathrm{I}_{h}$ (infinite cluster) and found that the lattice constant differs only $0.24 \AA$ from a $(\sqrt{3} \times \sqrt{3})$ $\mathrm{R} 30^{\circ}$ graphene supercell, which compares well with the difference of $0.23 \AA$ found in Ref. 4. Therefore, we are allowed to use this supercell to examine the graphene-ice interaction. We found that the adsorption of ice on graphene has also a minor influence on the geometry in the ice layer.

\section{B. Binding/adsorption energy}

The minor changes in the shape of the water cluster after adsorption on graphene indicate that the binding energy in the cluster is much larger than the adsorption energy. To make this statement more quantitative, we calculate both energies.

The adsorption energy of the water clusters on graphene is defined as $E_{a}=\left(E_{\text {cluster }}+E_{\text {graph }}\right)-E_{\text {tot }}$, where $E_{\text {cluster }}$ is given by the energy of the separately relaxed cluster, $E_{\text {graph }}$ by the energy of a perfect graphene layer, and $E_{\text {tot }}$ by the total energy of the relaxed interacting but relaxed cluster-graphene system. The energy related with the dipole interactions between water molecules of different supercells does not change much after adsorption on graphene. Consequently, this interaction energy will largely cancel in $E_{\text {tot }}$ and can, therefore, be neglected.

The resulting adsorption energy for the different clustergraphene systems is given in Table I. Both the total adsorption energy (per cluster) and the average adsorption energy per molecule are given. The total adsorption energy becomes larger for larger water clusters, which is expected, and the adsorption energy per molecule tends to converge to a smaller value of approximately $13 \mathrm{meV}$ for large clusters. Ice is bounded a little stronger than the water clusters. However, all the adsorption energies are small, implying that water 
TABLE I. $\mathrm{H}_{2} \mathrm{O}$ on graphene: the total adsorption energy $\left(E_{a}\right)$ per cluster and the average per molecule for different-size clusters and for different orientations.

\begin{tabular}{lccc}
\hline \hline No. of $\mathrm{H}_{2} \mathrm{O}$ mol. & Orientation & $E_{a}(\mathrm{eV})$ & $E_{a}(\mathrm{eV}) / \mathrm{mol}$. \\
\hline 1 & $a$ & 0.021 & 0.021 \\
1 & $b$ & 0.029 & 0.029 \\
2 & $a$ & 0.028 & 0.014 \\
2 & $b$ & 0.065 & 0.032 \\
2 & $c$ & 0.056 & 0.028 \\
3 & $a$ & 0.048 & 0.016 \\
3 & $b$ & 0.049 & 0.016 \\
4 & & 0.052 & 0.013 \\
5 & $a$ & 0.064 & 0.013 \\
5 & $b$ & 0.065 & 0.013 \\
2 (ice) & $a$ & 0.027 & 0.013 \\
2 (ice) & $b$ & 0.030 & 0.015 \\
\hline \hline
\end{tabular}

clusters are only weakly bound to perfect graphene sheets. One can also notice that clusters with more $\mathrm{H}-\mathrm{O}$ bonds pointing to the graphene surface tend to bind more strongly than those with $\mathrm{H}-\mathrm{O}$ bonds pointing away from the surface. ${ }^{5,22}$ However, for larger clusters, the relative difference between the number of hydrogen bonds pointing up or down becomes small. This makes the influence of the orientation of a large cluster on the adsorption energy small, suggesting that both orientations are likely to occur. Notice that these energies are of the order of room temperature $(26 \mathrm{meV})$, so our calculations are only applicable to real systems at low temperatures.

For the calculation of the binding energy of the $\mathrm{H}_{2} \mathrm{O}$ molecules in a water cluster, we used a supercell with the same dimensions as a $4 \times 4$ graphene supercell. But when calculating these binding energies, the energy associated with the dipole interactions between different supercells does not cancel anymore. However, their relative importance is now much lower because the energies associated with hydrogen bonding are larger. As a consequence, we can neglect this dipole interaction again. The results for the total binding energy and the average binding energy per molecule are given in Table II. The binding energy increases as the clusters grow because more hydrogen bonds can be formed and the angles between the different $\mathrm{H}$ bonds in larger clusters approach those of the ideal tetrahedral bond angles in ice structures.

A comparison of Tables I and II makes clear that the binding energies are, indeed, an order of magnitude larger than

TABLE II. $\mathrm{H}_{2} \mathrm{O}$ on graphene: the total binding energy $\left(E_{b}\right)$ per cluster and the average per molecule for different-size clusters.

\begin{tabular}{lcc}
\hline \hline No. of $\mathrm{H}_{2} \mathrm{O}$ mol. & $E_{b}(\mathrm{eV})$ & $E_{b}(\mathrm{eV}) / \mathrm{mol}$. \\
\hline 2 & 0.24 & 0.12 \\
3 & 0.71 & 0.24 \\
4 & 1.28 & 0.32 \\
5 & 1.70 & 0.34 \\
2 (ice) & 0.92 & 0.46 \\
\hline \hline
\end{tabular}

TABLE III. Charge transfers: the CT from graphene to the water molecules and the average CT per molecule for different-size clusters and for different orientations.

\begin{tabular}{lcrc}
\hline \hline No. of $\mathrm{H}_{2} \mathrm{O}$ mol. & Orientation & $\mathrm{CT}(\mathrm{e})$ & $\mathrm{CT}(\mathrm{e}) / \mathrm{mol}$. \\
\hline 1 & $a$ & -0.0028 & -0.0028 \\
1 & $b$ & 0.0098 & 0.0098 \\
2 & $a$ & 0.0026 & 0.0013 \\
2 & $b$ & 0.0114 & 0.0057 \\
2 & $c$ & 0.0073 & 0.0036 \\
3 & $a$ & 0.0038 & 0.0013 \\
3 & $b$ & 0.0114 & 0.0038 \\
4 & & 0.0058 & 0.0014 \\
5 & $a$ & 0.0065 & 0.0013 \\
5 & $b$ & 0.0094 & 0.0019 \\
2 (ice) & $a$ & -0.0016 & -0.0008 \\
2 (ice) & $b$ & 0.0064 & 0.0032 \\
\hline \hline
\end{tabular}

the associated adsorption energies. This indicates that water molecules will always form clusters on a graphene sheet or, stated differently, graphene is strongly hydrophobic.

\section{Charge transfer}

Using an extended version of the Hirshfeld chargeanalysis method ${ }^{6,23,24}$ we calculated the charge transfer between the water clusters and graphene. The results are given in Table III.

The average charge transfer per molecule of a cluster decreases as the cluster gets larger and converges to a value of approximately $0.002 e$, transferred from graphene to the molecules. All the found charge transfers are quite small, and it is doubtful whether these will have any substantial influence on the electronic properties of graphene. The reason the interaction between perfect graphene and water is so small is probably due to the absence of any molecular orbital of the clusters close to the Fermi level. ${ }^{4}$ We have calculated the density of states for the most stable orientation of the clusters on graphene and found that the molecular orbitals of the water molecules are, indeed, located far away from the Dirac point of graphene: for the different clusters, the highest occupied molecular orbitals are located between 2.3 and $3.2 \mathrm{eV}$ below the Dirac point and the lowest unoccupied molecular orbitals are located between 2.9 and $3.2 \mathrm{eV}$ above the Dirac point (see Fig. 2). To conclude, the charge transfer between intrinsic graphene and water clusters is negligible and will have no influence on the electronic properties of graphene.

\section{Electric-dipole moments}

Recently, Wehling et al. $^{4}$ suggested that the dipole moment of the water molecules on graphene might have a crucial influence on the energy shift of the impurity bands of an underlying $\left(\mathrm{SiO}_{2}\right)$ substrate, putting them closer to the Dirac point of graphene and resulting in a doping of the graphene layer. Therefore, it is interesting to know how the dipole moments of the different clusters are oriented. We calculated 


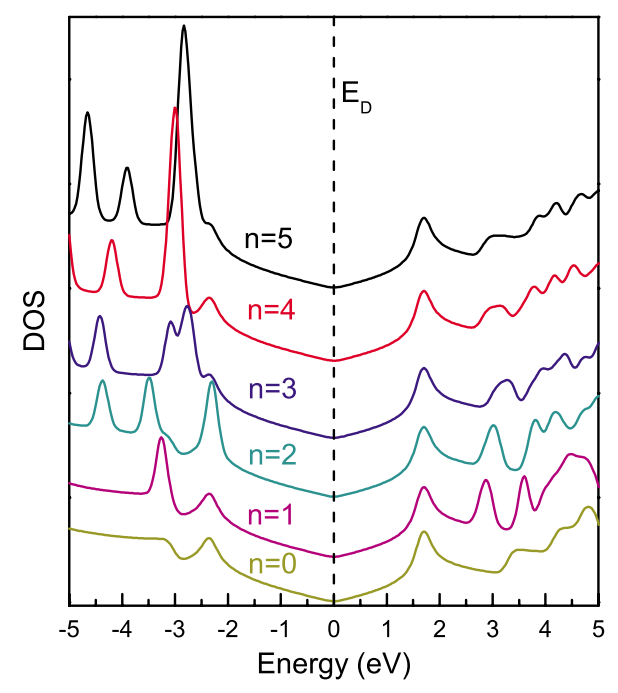

FIG. 2. (Color online) The total density of states of the different water clusters on graphene in the most stable orientations. The number of water molecules in the cluster is denoted by $n$.

the total strength of the dipoles and also their projections, perpendicular and parallel to the graphene surface. The results for the different clusters are given in Table IV.

The dipole moments of neighboring molecules in the same cluster are directed as much as possible in opposite directions. As a consequence, the average dipole moment per molecule in large clusters will become vanishingly small (see Table IV). Only in the case when the concentration of water molecules becomes large enough in order to form ice layers, will an appreciable dipole moment appear. The reason for this is clear: because water molecules in icelike structures have more than two neighboring molecules with which they form hydrogen bonds, they have less freedom in their orientation and are not able to point their dipole moments in opposite directions. As a result, the dipole moments of the different molecules accumulate and form a dipole layer on graphene. Such dipole moments, which have an influence on the electronic properties of graphene, ${ }^{4}$ have been observed experimentally. ${ }^{25}$ But for lower concentration, there will be no direct influence of the electric-dipole moment of relatively small water clusters on graphene. However, because of the large dielectric constant of water $\epsilon_{w}=80$, the water clusters can indirectly influence the electronic properties of graphene through, e.g., the screening of the charge-charge interaction and the screening of the Coulomb interaction of ionized impurities that are situated in graphene and/or in the substrate that supports the graphene layer. ${ }^{2,26}$
TABLE IV. Dipole moments: the total dipole moment $(P)$ as well as the dipole moment parallel $\left(P_{\|}\right)$and perpendicular $\left(P_{\perp}\right)$ to the graphene sheet for water clusters with different sizes and orientations $\left(P_{\perp}\right.$ is positive when pointing away from the sheet and negative when pointing to the sheet). The average dipole perpendicular to graphene per cluster is also shown. All dipoles are given in Debye (D) units.

\begin{tabular}{lcccrr}
\hline \hline No. of $\mathrm{H}_{2} \mathrm{O}$ mol. & Orient. & $P$ & $P_{\|}$ & \multicolumn{1}{c}{$P_{\perp}$} & $P_{\perp} / \mathrm{mol}$. \\
\hline 1 & $a$ & 1.80 & 0.00 & 1.80 & 1.80 \\
1 & $b$ & 1.82 & 1.36 & -0.66 & -0.66 \\
2 & $a$ & 2.94 & 2.94 & -0.11 & -0.05 \\
2 & $b$ & 2.87 & 2.87 & 0.10 & 0.05 \\
2 & $c$ & 2.66 & 2.66 & -0.09 & -0.05 \\
3 & $a$ & 1.24 & 0.03 & -1.24 & -0.41 \\
3 & $b$ & 1.15 & 0.04 & 1.15 & 0.38 \\
4 & & 0.03 & 0.03 & -0.02 & -0.01 \\
5 & $a$ & 1.08 & 0.18 & -1.06 & -0.21 \\
5 & $b$ & 1.00 & 0.24 & 0.97 & 0.19 \\
2 (ice) & $a$ & $/$ & $/$ & 1.62 & 0.81 \\
2 (ice) & $b$ & $/$ & $/$ & -1.63 & -0.81 \\
\hline \hline
\end{tabular}

\section{CONCLUSIONS}

We studied the influence of water clusters on the electronic properties of a perfect graphene sheet using $a b$ initio DFT calculations. The binding energy of the water molecules in a cluster is an order of magnitude larger than the adsorption energy between the cluster and graphene, indicating that graphene is very hydrophobic. Charge transfers between a graphene sheet and water clusters, which could influence the density of the charge carriers and, consequently, the resistance in graphene, are very small. The molecules in a water cluster tend to orient their dipole moments in opposite directions, so that they cancel on average. But in the limit of a large concentration of water molecules such that icelike structures can be formed, the dipole moments of the different molecules accumulate instead of averaging out, leading to an appreciable total dipole moment that will have an influence on the electronic properties of graphene. ${ }^{4}$

\section{ACKNOWLEDGMENTS}

This work was supported by the Flemish Science Foundation (FWO-V1), the NOI-BOF of the University of Antwerp, and the Belgian Science Policy (IAP).

\footnotetext{
*ortwin.leenaerts@ua.ac.be

†bart.partoens@ua.ac.be

*francois.peeters@ua.ac.be

${ }^{1}$ K. S. Novoselov, A. K. Geim, S. V. Morozov, D. Jiang, Y. Zhang, S. V. Dubonos, I. V. Grigorieva, and A. A. Firsov, Sci-
}

ence 306, 666 (2004)

${ }^{2}$ F. Schedin, A. K. Geim, S. V. Morozov, E. W. Hill, P. Blake, M. I. Katsnelson, and K. S. Novoselov, Nature Mater. 6, 652 (2007).

${ }^{3}$ T. O. Wehling, K. S. Novoselov, S. V. Morozov, E. E. Vdovin, 
M. I. Katsnelson, A. K. Geim, and A. I. Lichtenstein, Nano Lett. 8, 173 (2008)

${ }^{4}$ T. O. Wehling, A. I. Lichtenstein, and M. I. Katsnelson, Appl. Phys. Lett. 93, 202110 (2008).

${ }^{5}$ O. Leenaerts, B. Partoens, and F. M. Peeters, Phys. Rev. B 77, 125416 (2008).

${ }^{6}$ O. Leenaerts, B. Partoens, and F. M. Peeters, Appl. Phys. Lett. 92, 243125 (2008).

${ }^{7}$ F. Cervantes-Sodi, G. Csanyi, S. Piscanec, and A. C. Ferrari, Phys. Rev. B 77, 165427 (2008).

${ }^{8}$ J. Sabio, C. Seoanez, S. Fratini, F. Guinea, A. H. Castro Neto, and F. Sols, Phys. Rev. B 77, 195409 (2008).

${ }^{9}$ B. Huang, Z. Y. Li, Z. R. Liu, G. Zhou, S. G. Hao, J. Wu, B. L. Gu, and W. H. Duan, J. Phys. Chem. C 112, 13442 (2008).

${ }^{10}$ Z. M. Ao, J. Yang, S. Li, and Q. Jiang, Chem. Phys. Lett. 461, 276 (2008)

${ }^{11}$ J. P. Perdew, K. Burke, and M. Ernzerhof, Phys. Rev. Lett. 77, 3865 (1996)

${ }^{12}$ D. R. Hamann, Phys. Rev. B 55, R10157 (1997).

${ }^{13}$ T. Kurita, S. Okada, and A. Oshiyama, Phys. Rev. B 75, 205424 (2007).

${ }^{14}$ A. Maiti, J. Andzelm, N. Tanpipat, and P. von Allmen, Phys. Rev. Lett. 87, 155502 (2001)
${ }^{15}$ X. Gonze, J.-M. Beuken, R. Caracas, F. Detraux, M. Fuchs, G.-M. Rignanese, L. Sindic, M. Verstraete, G. Zerah, F. Jollet, M. Torrent, A. Roy, M. Mikami, Ph. Ghosez, J.-Y. Raty, and D. C. Allan, Comput. Mater. Sci. 25, 478 (2002).

${ }^{16} \mathrm{M}$. Torrent, F. Jollet, F. Bottin, G. Zerah, and X. Gonze, Comput. Mater. Sci. 42, 337 (2008)

${ }^{17}$ H. J. Monkhorst and J. D. Pack, Phys. Rev. B 13, 5188 (1976).

${ }^{18}$ S. S. Xantheas and T. H. Dunning, J. Chem. Phys. 99, 8774 (1993).

${ }^{19}$ F. K. Keutsch and R. J. Saykally, Proc. Natl. Acad. Sci. U.S.A. 98, 10533 (2001).

${ }^{20}$ S. Maheshwary, N. Patel, N. Sathyamurthy, A. D. Kulkarni, and S. R. Gadre, J. Phys. Chem. A 105, 10525 (2001).

${ }^{21}$ J. Berashevich and T. Chakraborty, arXiv:0901.4956 (unpublished).

${ }^{22}$ M. C. Gordillo and J. Marti, Phys. Rev. B 78, 075432 (2008).

${ }^{23}$ F. L. Hirshfeld, Theor. Chim. Acta 44, 129 (1977).

${ }^{24}$ P. Bultinck, C. Van Alsenoy, P. W. Ayers, and R. Carbo-Dorca, J. Chem. Phys. 126, 144111 (2007).

${ }^{25}$ J. Moser, A. Verdaguer, D. Jiménez, A. Barreiro, and A. Bachtold, Appl. Phys. Lett. 92, 123507 (2008).

${ }^{26}$ C. Jang, S. Adam, J.-H. Chen, E. D. Williams, S. Das Sarma, and M. S. Fuhrer, Phys. Rev. Lett. 101, 146805 (2008). 